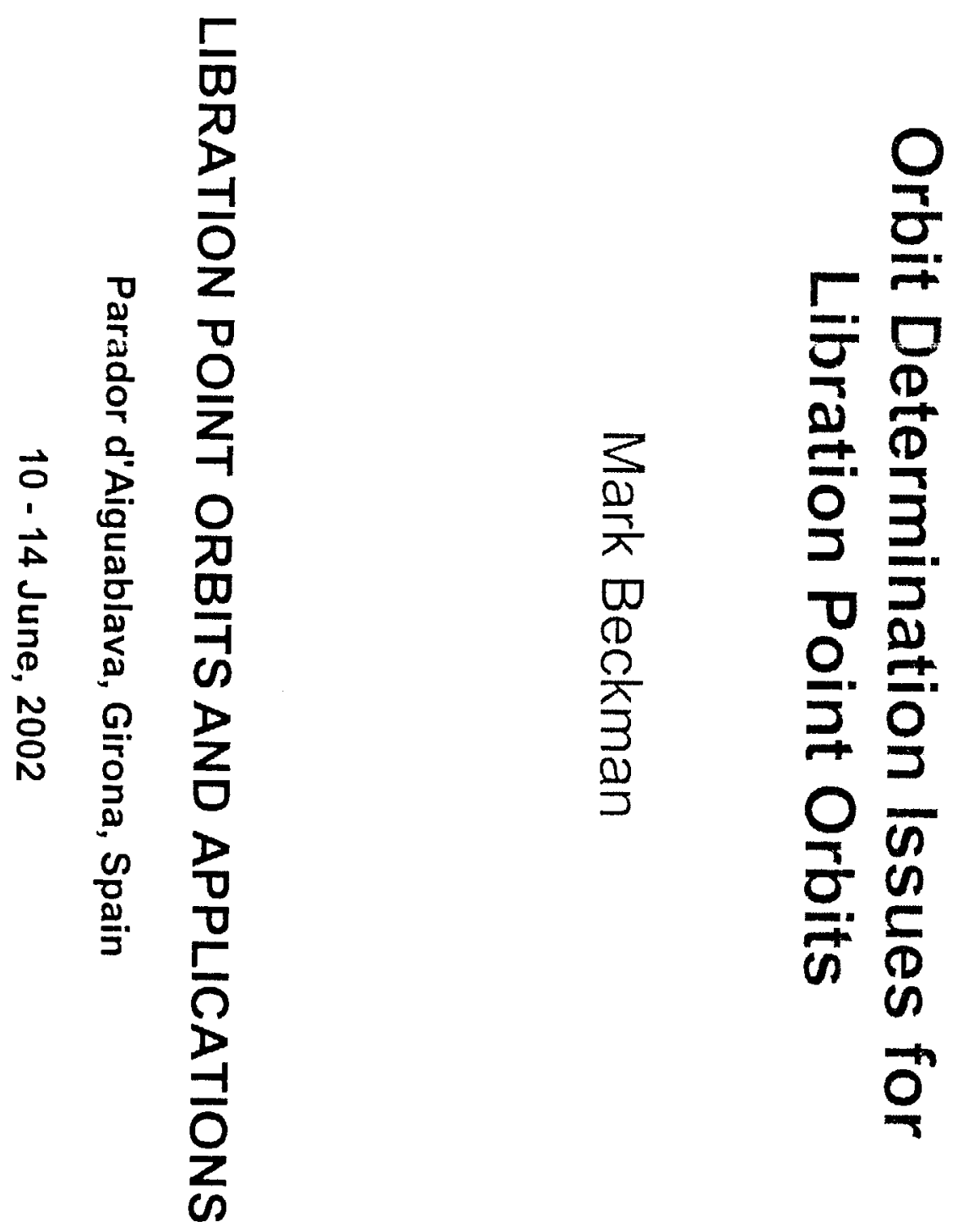




\title{
Orbit Determination Issues for Libration Point Orbits
}

\author{
Mark Beckman \\ Guidance, Navigatic a \& Control Center’s Flight Dynamics Analysis Branch (Code 572) \\ NASA s Goddard Space Flight Center, Greenbelt, MD 20771 \\ -301-286-8866
}

\begin{abstract}
Libration point mission designers require knowledge of orbital accuracy for a variety of analyses including station kee ing control strategies, transfer trajectory design, and formation and constellation control. Past pub ications have detailed orbit determination (OD) results from individual libration point missions. This f aper collects both published and unpublished results from four previous libration point missions (ISEE 3, SOHO, ACE and MAP) supported by Goddard Space Flight Center's Guidance, Navigation \& Control Center. The results of those missions are presented along with OD issues specific to each mission. All p. st missions have been limited to ground based tracking through NASA ground sites using standard rar ge and Doppler measurement types. Advanced technology is enabling other OD options including onboard navigation using onboard attitude sensors and the use of the Very Long Baseline Interferometry (VLB) measurement Delta Differenced One-Way Range (DDOR). Both options potentially enable missions to :educe coherent dedicated tracking passes while maintaining orbital accuracy. With the increased pojected loading of the DSN, missions must find alternatives to the standard OD scenario.
\end{abstract}

\section{INTRODUCTION}

Orbit determination for librition point orbits (LPOs) is quite unique. The regime is far from the Low Earth Orbits (LEO) t pically supported by the Goddard Space Flight ( enter (GSFC) and far from the interplanetary orbits supported by the Jet Propulsion Laboratory JPL). The regime offers very little dynamic: thus requiring extensive amount of time and tracking data in order to attain a solution.

NASA's GSFC has designe 1 and supported every libration point mission except the recent Genesis mission out of JPL. T wis paper presents a summary of the anal ses and orbit determination results of four revious libration point missions supported by ('SFC's Guidance, Navigation \& Control Center (GNCC). ISEE-3 was the first libration point mission in 1978. ISEE-3 stayed in a halo orbit for only three years before departing on the next ph ise of its mission. It was 17 years later before a second mission, $\mathrm{SOHO}$, was flown to a libration point. SOHO was the first mission designed to remain in the vicinity of a libration for the mission duration. $\mathrm{SOHO}$ was quickly followed $\mathrm{h} \mathrm{ACE}$, the first mission to follow the quasi-priodic Lissajous orbit pattern. The latest missio ?, MAP, the first mission to the Earth-Sun L.: point, is also presented here with as yet unpulished results.
The majority of these missions have used the Deep Space Network (DSN) assets to support tracking services. However, with the projected loading on the DSN in future years, this option is becoming far less feasible. Alternatives include commercial tracking assets such as the Universal Space Network (USN), which is scheduled to support the future Triana mission, and the use of advanced technology to reduce the required tracking services from the DSN.

One such technology is the use of Celestial Navigator (CelNav). CelNav is a onboard Kalman filter that processes one-way forward Doppler measurements and onboard attitude sensor data. CelNav analysis results are presented here.

Another altemative is the use of the Very Long Baseline Interferometry (VLBI) measurement called Delta Differenced One-Way Range (DDOR). DDOR is actually an angular measurement from a nearby quasar to the spacecraft. DDOR is being implemented at some of the DSN sites and its applicability to LPOs is presented here. 


\section{PAST MISSIONS}

International Sun-Earth Exylorer-3 (ISEE-3)

The first libration point $\mathrm{mission}$ was ISEE-3 (Figure 1). Launched as part of an international cooperative agreement betwern ESA and NASA on August 12, 1978, ISEE-3 cntered a large halo orbit about the Earth-Sun L1 point on November 20,1978 . The spacecraft remained in the halo orbit for $31 / 2$ years before deprarting on June 10 , 1982 for the second phase of its mission.

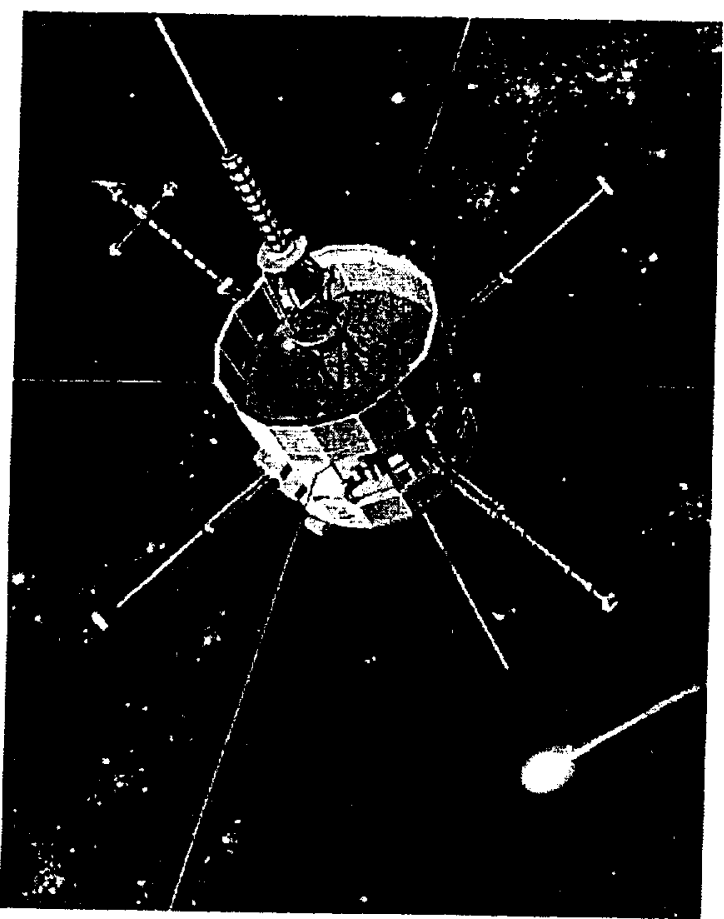

Figure 1: ISEE-3 Spacecraft

ISEE-3 flew in a large halo o bit about $\mathrm{Ll}$ of approximately $600,000 \mathrm{~km}$ in $\mathrm{Y}$-amplitude (in ecliptic plane perpendicular to Earth-Sun line). The dynamics in the vicinity of the libration point are not significantly diffe ent for different size halo or Lissajous orbits. All have an approximate period of 6 morths. The class, phase and Z-amplitude (out of the ecliptic) of the halo or Lissajous orbit all have an effect on the orbital accuracy. However, these effects are small and, given the number of variables affecting orbital accuracy, it is u iually measured only to order-of-magnitude.

ISEE-3 was ground tracked by NASA S-band Tracking Data Network (STDN) sites during the halo orbit phase. The tracking schedule was irregular but generally consist d of multiple short passes ( 5 minutes) at acquisition-of-signal (AOS), maximum elevation, and near loss-ofsignal (LOS) from each station [Joyce 84]. Covariance analysis was performed pre-mission to assess the expected orbital uncertainties. Covariance analysis indicated an optimum batch tracking arc length of 21 days. Station-keeping maneuvers were performed every 45 days enabling two completely independent orbital solutions between each maneuver. $O D$ was performed every other week giving a 7-day overlap period. The covariance analysis was comparable to comparisons between consecutive definitive solutions obtained during the actual mission. The definitive overlap comparisons are obtained by differencing the trajectories obtained by the two overlapping solutions. Table 1 details the covariance analysis and definitive overlap comparisons.

Table 1: ISEE-3 Comparisons of Overlap Differences and Covariance Analysis [Joyce 84]

\begin{tabular}{|c|c|c|}
\hline Period & $\begin{array}{c}\text { Overlap } \\
\text { Compare }(\mathrm{km})\end{array}$ & $\begin{array}{c}\text { Covariance } \\
\text { Analysis }(\mathrm{km})\end{array}$ \\
\hline A & 8.1 & 6.0 \\
\hline B & 9.0 & 5.5 \\
\hline C & 3.6 & 5.4 \\
\hline
\end{tabular}

Corresponding velocity uncertainties were 0.3 to $2.0 \mathrm{~cm} / \mathrm{sec}$ from the definitive overlap differences.

Definitive overlap comparisons are not a direct measure of absolute orbital accuracy. However, without an independent tracking source, they are the best available measure.

\section{Solar \& Heliospheric Observatory (SOHO)}

SOHO was launched on December 2, 1995 as a joint ESA and NASA mission. SOHO performed a direct insertion into a large Earth-Sun $L 1$ halo orbit with a $\mathrm{Y}$-amplitude of approximately $670,000 \mathrm{~km}$ (see Figure 2).

SOHO tracking is performed by the DSN, primarily the 26-m antennas, but some $34-\mathrm{m}$ and 70-m MARK IVA antennas are also used. The MARK IVA SRA ranging system is generally slightly more accurate. The nominal tracking schedule for SOHO is 5 hours per day from alternating DSN sites. This schedule is extremely inconsistent for SOHO however. 

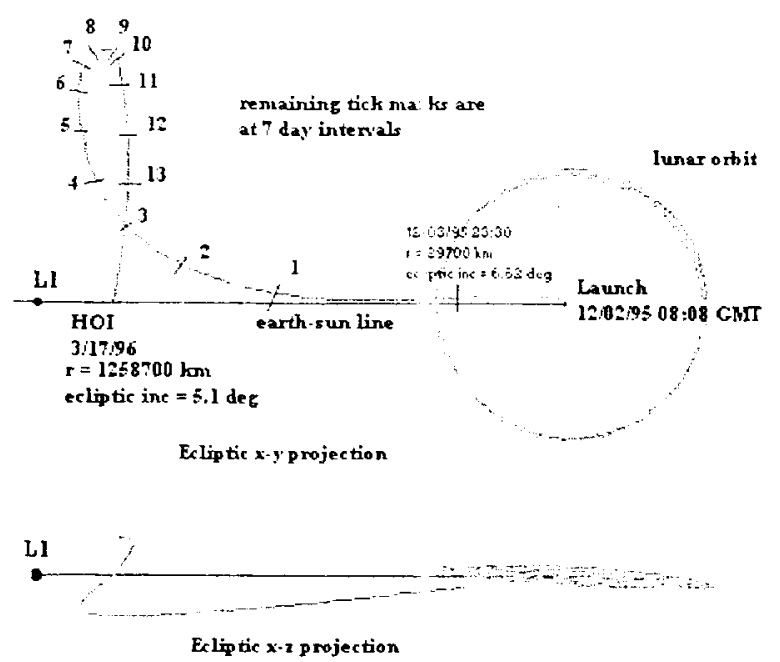

Figure 2: SOHO Trajectory in Solar Rotating Coordinats

Covariance analysis was pertomed pre-mission in order to assess orbital accuracy and to deternine the batch arc leng $h$. The covariance analysis used a conservative tiacking schedule of only 1 hour per day. This analysis indicated that an optimum arc length of 21 days would give orbital accuracy to less tha $9 \mathrm{~km}$. Table 2 details these results.

Table 2: SOHO Covariance Analysis Results [Jordan 93]

\begin{tabular}{|c|c|c|c|c|c|}
\hline $\begin{array}{c}\text { Data } \\
\text { Span } \\
\text { (d) }\end{array}$ & Axis & \multicolumn{3}{|c|}{ Maximu } & \multicolumn{2}{c|}{ Total Error } \\
\cline { 3 - 6 } & & $\begin{array}{c}\text { Def Period } \\
\text { Pos } \\
(\mathrm{km})\end{array}$ & $\begin{array}{c}\text { Vel } \\
(\mathrm{cm} / \mathrm{s})\end{array}$ & $\begin{array}{c}\text { 200d Pos } \\
(\mathrm{km})\end{array}$ & $\begin{array}{c}\text { Ved } \\
(\mathrm{cm} / \mathrm{s})\end{array}$ \\
\hline 14 & $\mathrm{Y}$ & 10.1 & 0.42 & 17.4 & 0.79 \\
\hline 21 & $\mathrm{Y}$ & 8.6 & 0.19 & 8.4 & 0.34 \\
\hline 14 & $\mathrm{Z}$ & 6.6 & 0.39 & 20.1 & 1.05 \\
\hline 21 & $\mathrm{Z}$ & 5.7 & 0.26 & 11.3 & 0.06 \\
\hline
\end{tabular}

SOHO performs station-kesping maneuvers every 8 to 12 weeks. Additionally, attitude maneuvers are performed much more frequently with the use of spacecraft thiusters. While the attitude maneuvers are designell for zero delta- $V$, thruster performance and misalignments contribute about a $5 \%$ error. Tle batch definitive arcs are broken at all maneuve: points instead of attempting to model these mareuvers. Modeling would add an additional erro source into the solution and would require: detailed engine model in the OD software. Data arcs were generally kept at the standar 21 days when possible, but were often shortt $r$. As part of the solution process, the solar radiation pressure coefficient $\left(C_{r}\right)$ was estimated along with range biases for each pass from the MARK IVA antennas (averaging about 6 per solution).

SOHO's definitive orerlap requirements were 50 $\mathrm{km}$ and $3 \mathrm{~cm} / \mathrm{sec}$. During long periods free of spacecraft perturbations, overlap comparisons were obtained. Actual definitive overlap comparisons average about $7 \mathrm{~km}$. That uncertainty is primarily in the cross-track direction (plane-of-the-sky perpendicular to the projection of the velocity vector into that plane). Radial uncertainty are generally less than $1 \mathrm{~km}$. Table 3 details the position and velocity definitive overlap comparisons.

Table 3: SOHO Definitive Overlap Comparisons

\begin{tabular}{|c|c|c|c|c|}
\hline & $\underline{\text { RSS }}$ & $\underline{\text { Radial }}$ & $\frac{\text { Along- }}{\text { track }}$ & $\frac{\underline{\text { Cross- }}}{\text { track }}$ \\
\hline $\begin{array}{c}\text { Pos } \\
(\mathrm{km})\end{array}$ & 7 & 1 & 2 & 7 \\
\hline $\begin{array}{c}\text { Vel } \\
(\mathrm{mm} / \mathrm{s})\end{array}$ & 0.4 & 0.1 & 0.2 & 0.3 \\
\hline
\end{tabular}

These overlap compares were obtained during long periods without spacecraft perturbations. The routine OD for SOHO was not typically this accurate due to the use of much shorter data arcs.

The predicted orbital uncertainty requirement after a 44-day propagation is $100 \mathrm{~km}$ and 10 $\mathrm{cm} / \mathrm{sec}$. Definitive solutions were compared to predicted solutions after 44 days of propagation to obtain a predictive overlap comparison. The SOHO predictive overlaps were generally around $14 \mathrm{~km}$. Table 4 details the predictive overlap comparisons. Note that the radial component is no longer constrained by the measurement data and grows significantly.

Table 4: SOHO Predictive Overlap Comparisons

\begin{tabular}{|c|c|c|c|c|}
\hline & $\underline{\text { RSS }}$ & $\underline{\text { Radia! }}$ & $\frac{\underline{\text { Along- }}}{\text { track }}$ & $\frac{\underline{\text { Cross- }}}{\text { track }}$ \\
\hline $\begin{array}{c}\text { Pos } \\
(\mathrm{km})\end{array}$ & 14 & 9 & 2 & 11 \\
\hline $\begin{array}{c}\mathrm{Vel} \\
(\mathrm{mm} / \mathrm{s})\end{array}$ & 0.7 & 0.2 & 0.3 & 0.6 \\
\hline
\end{tabular}

An additional study was performed for the MAP mission using real SOHO tracking data. This analysis was performed to show the effects of reducing the 5 hours per day of SOHO tracking data to only 37 minutes per day for MAP. The 
SOHO definitive ephemeris using all available tracking data was used as tie truth ephemeris. Table 5 shows the comparis ins for the reduced tracking data solutions. The r sults are somewhat erratic bul generally show a degradation of accuracy of less than $2 \mathrm{~km}$.

Table 5: SOHO Reduced Tracking Data Results [Nichols on 99]

\begin{tabular}{|c|c|c|c|c|}
\hline Epoch & Editting & $\mathrm{C}$ & $\begin{array}{c}\text { Pos } \\
\text { RSS } \\
(\mathrm{km})\end{array}$ & $\begin{array}{c}\text { Vel } \\
\text { RSS } \\
(\mathrm{cm} / \mathrm{s} \\
\mathrm{ec})\end{array}$ \\
\hline 980111 & None & 1.3 & $\mathrm{NA}$ & $\mathrm{NA}$ \\
\hline 980111 & $37 \mathrm{~min} /$ day & 1.3 & 8.31 & 0.236 \\
\hline 980111 & $\begin{array}{c}37 \mathrm{~min} \\
\text { twice/day }\end{array}$ & 1.36 & 0.71 & 0.081 \\
\hline 980321 & None & 1.36 & $\mathrm{NA}$ & $\mathrm{NA}$ \\
\hline 980321 & $37 \mathrm{~min} /$ day & 1.2 & 19.6 & 3.82 \\
\hline 980405 & None & 1.3 & $\mathrm{NA}$ & $\mathrm{NA}$ \\
\hline 980405 & $37 \mathrm{~min} /$ day & 1.360 & 0.65 & 0.142 \\
\hline 980417 & None & 1.38 & $\mathrm{NA}$ & $\mathrm{NA}$ \\
\hline 980417 & $37 \mathrm{~min} /$ day & 1.39 & 1.21 & 0.411 \\
\hline
\end{tabular}

\section{Advanced Composition Explored (ACE)}

ACE was launched on August 25, 1997 as a NASA Explorer program mission. ACE performed a direct insertion in:o an Earth-Sun L1 small Lissajous orbit with is Y-amplitude of about $150,000 \mathrm{~km}$ (see Figure 3). ACE was the first spacecraft to fly in the quasi-periodic Lissajous pattern. The periodic halo orbits do not exist at the smaller amplitudes.

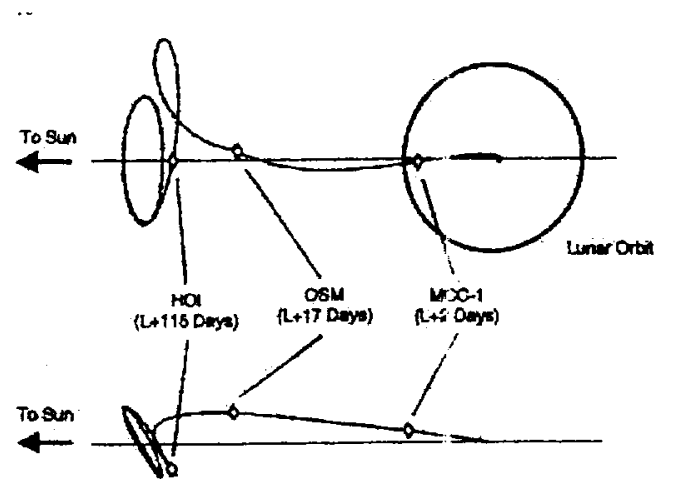

Figure 4: ACE Trajectory in Solar Rotating Coordinates

The ACE spacecraft is spin-stibilized at $5 \mathrm{rpm}$ with the spin axis of the spacecraft required to point within 20 degrees of the $S \mathrm{~m}$ at all times. In addition, the High Gain Antenna (HGA) is required to point Earth-ward within 4.5 degrees. These two constraints require $A C E$ to perform reorientation maneuvers as frequently as every 5 days. These maneuvers are performed with thrusters and therefore force the analysts to break the arc around these maneuvers to obtain clean data arcs free of spacecraft perturbations. Thus, ACE uses data arcs of 4 to 14 days, which are clearly not optimal for OD accuracy [Colombe $02]$. For the longer data arcs, $C_{T}$ and pass dependent range biases from $70-\mathrm{m}$ sites are estimated.

ACE gets approximately one 3.5-hour pass per day from the DSN with an additional 2 or 3 onehour passes per week. The DSN data is primarily from the 26-m and 34-m sites.

Because ACE extends the batch data arc as long as possible between attitude maneuvers, there are no definitive overlap comparisons available. Single point overlaps are obtained by differencing consecutive definitive solutions at the time of the attitude maneuvers. Those overlaps indicate a mean position difference of $10 \mathrm{~km}$ and a velocity difference of $1.2 \mathrm{~cm} / \mathrm{sec}$. Table 6 details the ACE overlap conparisons.

Table 6: ACE Overlap Comparisons

\begin{tabular}{|c|c|c|c|c|}
\hline & $\underline{\text { RSS }}$ & $\underline{\text { Radial }}$ & $\frac{\text { Along- }}{\text { track }}$ & $\underline{\underline{\text { Cross- }}}$ \\
\hline \multicolumn{5}{|c|}{ Definitive Point Overlap } \\
\hline $\begin{array}{c}\text { Pos } \\
(\mathrm{km})\end{array}$ & 10 & 4 & 5 & 8 \\
\hline $\begin{array}{c}\text { Vel } \\
(\mathrm{mm} / \mathrm{s})\end{array}$ & 1.2 & 0.9 & 0.1 & 0.9 \\
\hline \multicolumn{5}{|c|}{$2-$ Week Predictive Overlap } \\
\hline $\begin{array}{c}\text { Pos } \\
(\mathrm{km})\end{array}$ & 23 & 8 & 6 & 21 \\
\hline $\begin{array}{c}\mathrm{Vel} \\
(\mathrm{mm} / \mathrm{s})\end{array}$ & 1.2 & 0.9 & 0.1 & 0.9 \\
\hline
\end{tabular}

ACE never attempted to model the spacecraft maneuvers in order to obtain longer tracking arcs. Analysis was done for this particular scenario for a future libration point mission, Constellation-X. At the time, the current Constellation-X design called for momentum unloads using spacecraft thrusters every other day. Since a two-day arc was clearly not sufficient for $O D$, modeling of the maneuvers would be required.

Covariance analysis was performed for a scenario with $4 \mathrm{~mm} / \mathrm{sec}$ delta-Vs applied every other day with a thruster performance uncertainty of either $3 \%$ or $5 \%$. The delta-VS were applied toward the Sun in hopes absorbing 
some of the error in the stimated $C_{r}$. The tracking schedule used was 1) minutes of range and Doppler tracking data very day from a single station with a 21 -day tracking data arc. Estimating the spacecraft muneuvers was nut possible due to the sheer numler.

The definitive $O D$ position and velocity errors for the $3 \%$ and $5 \%$ delta- $\mathrm{V}$ er or cases as shown in Table 7 .

Table 7: Constellation-X Co rariance Analysis Assuming Multiple Spacecraft Maneuvers Marr 98]

\begin{tabular}{|c|c|c|}
\hline Delta-V Error & $\begin{array}{c}\text { Pos Error } \\
(\mathrm{km})\end{array}$ & $\begin{array}{c}\text { Vel Error } \\
(\mathrm{cm} / \mathrm{sec})\end{array}$ \\
\hline $3 \%$ & $12-47$ & $3.5-4.0$ \\
\hline $5 \%$ & $16-78$ & $5.8-6.5$ \\
\hline
\end{tabular}

Note that the errors seen in the Constellation- $X$ analysis are considerably highly than that seen for ACE using shorter data ar $>$ s. In addition, the larger velocity errors would 1 equire much more frequent station-keeping mane uvers, and a higher station-keeping delta- $\mathrm{V}$ bucget, in order to maintain the Lissajous orbit.

\section{Microware Anisotropy Probe (MAP)}

MAP is the latest libration puint mission. MAP was launched on June 30, 2001 and used a lunar swingby to inse1t into a smill Lissajous orbit about the Earth-Sun L2 point (see Figure 5). MAP is the first mission to remain in the vicinity of the $\mathrm{L} 2$ point for an extender! period of time.

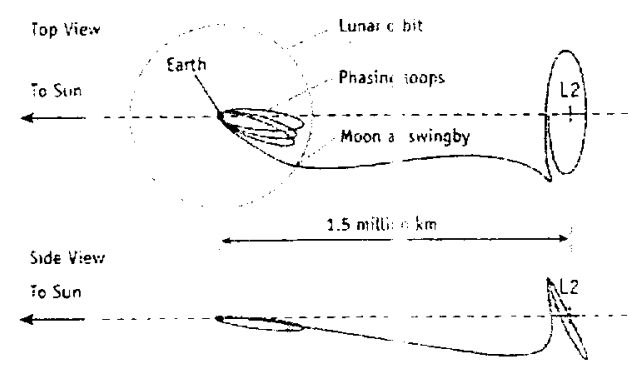

as:

Figure 5: MAP Trajectory in Solar Rotating Coordinate:

Tracking services for MAP are provided by the DSN. MAP receives a minmum of one 45 minute pass per day from the I)SN $34-\mathrm{m}$ or $70-\mathrm{m}$ sites. Because MAP receives txclusively MARK IVA tracking data, MAP has he highest quality measurement data set of any previous mission.
However, MAP does not possess an equivalent quantity of measurement data than earlier missions.

The MAP spacecraft is spin stabilized about an axis that precesses once per hour about a 22.5 . degree half-angle cone about the Sun-MAP line. Because of the unique attitude requirements for MAP, the cross-sectional area for solar radiation pressure forces is nearly constant. This greatly reduces attitude dependent errors on solar radiation force modeling which is typically a large error source. Most missions estimate the $C_{r}$ but current GSFC software limits the solar radiation force calculation to a fixed crosssectional area and a single constant estimated $C_{r}$ over the entire data arc. Thus, the estimated $C_{t}$ normally soaks up changing forces due to attitude changes and solar events. For MAP, this estimated $C_{r}$ is extremely consistent $( \pm 0.005)$ and varies only in response to solar events [Fink 02]. This improves overall OD accuracy for MAP.

Because of the $C_{r}$ consistency, MAP is able to use longer data arcs than other missions. MAP uses a minimum of 14-day arcs after maneuvers up to a current maximum of 72 days of spacecraft unperturbed motion.

In addition, because $\mathrm{MAP}$ receives a large amount of $70-\mathrm{m}$ tracking data, they have been able to calibrate the range biases from various stations and are able to apply these biases to future solutions. This eliminates numerous parameters from the estimated state vector [Fink 02].

Since OD data arcs are extended to much longer lengths for MAP, overlap differences do not exist. However, post-processed solutions using two consecutive 5-week arcs do give adequate comparisons. Over the short prediction span of 5 weeks, the overlap differences were $2.0 \mathrm{~km}$ and $0.83 \mathrm{~mm} / \mathrm{s}$. The overlaps increase when the prediction span is increased to 9 weeks: $6.7 \mathrm{~km}$ and $3.9 \mathrm{~mm} / \mathrm{s}$. Table 8 details the MAP results. 
Table 8: MAP Overlap Cornparisons |Fink

02]

\begin{tabular}{|c|c|c|c|c|}
\hline & $\underline{\mathrm{RSS}}$ & Radial & $\frac{\text { long- }}{\text { track }}$ & $\begin{array}{l}\text { Cross- } \\
\text { track }\end{array}$ \\
\hline \multicolumn{5}{|c|}{ 5-Week Predictive Jverlap } \\
\hline $\begin{array}{c}\text { Pos } \\
(\mathrm{km})\end{array}$ & 2.0 & 0.3 & 1.4 & 2.0 \\
\hline $\begin{array}{c}\text { Vel } \\
(\mathrm{mm} / \mathrm{s})\end{array}$ & 0.83 & 0.36 & 0.40 & 0.79 \\
\hline \multicolumn{5}{|c|}{ 9-Week Predictive Overlap } \\
\hline $\begin{array}{c}\text { Pos } \\
(\mathrm{km})\end{array}$ & 6.7 & 6.2 & 2.3 & 1.8 \\
\hline $\begin{array}{c}\text { Vel } \\
(\mathrm{m} m / \mathrm{s})\end{array}$ & 3.9 & 3.8 & 0.4 & 0.6 \\
\hline
\end{tabular}

These MAP results are fairly optimistic as they are taken during a period of relative solar inactivity and continuous science mode operation. Results are signifi:antly worse with irregularly high solar winds o: when the science mode attitude is changed.

\section{ADVANCED TECHNOLOCY}

\section{Celestial Navigator (CelNav)}

CelNav is a part of the Goddard Enhanced Onboard Navigation System (GEONS) software package developed by GSFC's GNCC. CelNav uses standard spacecraft attitude sensors and communication component; to provide autonomous navigation. A ralysis to date indicates that real-time autonomous navigation accuracies to $10 \mathrm{~km}$ RMS for LPO missions are achievable using high-accuracy attitude sensors and one-way Doppler measureinents [Folta 99].

CelNav uses directional mtasurements from standard attitude sensors (e.s. Earth and Sun sensors) and one-way foruard-link Doppler measurements from a ground station communications receiver ausmented with a Doppler extraction capability (see Figure 6 for a schematic). The one-way for ward Doppler is obtained from the spacecraft communication link, thus eliminating the nced for dedicated tracking services. The directional measurements are the angles of the line-of sight unit vector from the sensor to the celestial object, measured with respect to the sensor fram of reference.

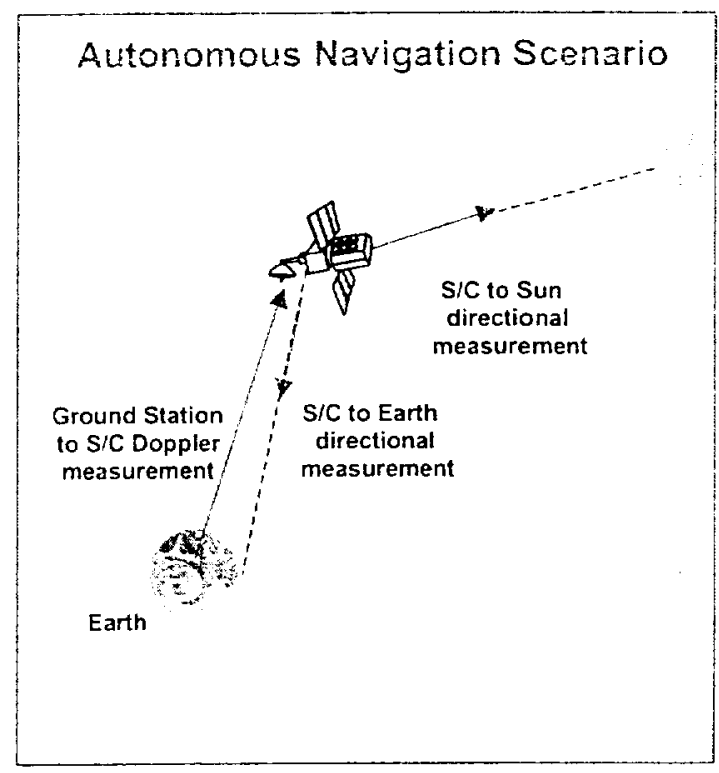

Figure 6: CelNav Measurement Sources

Simulated analyses using realistic and optimistic levels for the measurement noise and biases and the Doppler tracking frequency have been performed. Directional measurement noise standard deviations were selected to be consistent with the current digital sun sensor technology of 1 arc-minute and an onboard attitude determination accuracy of 1 arc-minute (achievable using star trackers). The one-way Doppler measurement accuracy is primarily dependent on the noise and stability characteristics of the onboard oscillator that provides the frequency reference used in the Doppler extraction process. The optimistic reference frequency quality was modeled based on expected performance of a typical ultra-stable oscillator (LSO) [Folta 99].

For the optimistic case using unbiased Earth and Sun directional measurements with noise consistent with current digital sun sensor technology and Doppler measurements referenced to an USO, orbital error was $7 \mathrm{~km}$ and $2 \mathrm{~mm} / \mathrm{sec}$. Various tracking scenarios are shown in Table 9. When Doppler tracking was eliminated, orbital errors increased significantly. The addition of more realistic parameters including a noise USO (10 times the noise sigma), reduced Doppler tracking data, directional measurement biases and the elimination of Earth directional measurements all degraded solution accuracy a range of 14 to 22 $\mathrm{km}$. 
Table 9: CelNav Solution Accuracy [Folta 99]

\begin{tabular}{|c|c|c|}
\hline Tracking Scenario & Po $\frac{\text { EIror }}{(\mathrm{km})}$ & $\begin{array}{l}\text { Vel Error } \\
\text { (mmisec) } \\
\end{array}$ \\
\hline Nominal & 7 & 2 \\
\hline $\begin{array}{l}\text { Eliminated Doppler } \\
\text { tracking }\end{array}$ & 62 & 30 \\
\hline $\begin{array}{l}\text { Increased Doppler } \\
\text { measurement noise } \\
\text { from } 0.001 \mathrm{~Hz} \text { to } 0.01 \\
\mathrm{~Hz}\end{array}$ & 22 & 3 \\
\hline $\begin{array}{l}\text { Reduced Doppler } \\
\text { tracking from } 2 \text { to } 14 \text { - } \\
\text { hr pass per day }\end{array}$ & 17 & $N A$ \\
\hline $\begin{array}{l}\text { Added directional } \\
\text { measurement bias of } \\
0.1 \text { arc-minute }\end{array}$ & 22 & 4 \\
\hline $\begin{array}{l}\text { Eliminated Earth } \\
\text { directional } \\
\text { measurements }\end{array}$ & 14 & NA \\
\hline
\end{tabular}

Steady-state accuracy was nut found to be very sensitive to elimination of Sun directional measurements or a 4-fold increase in the directional measurement noi e to 6 arc minutes (consistent with existing Earti sensor technology and 0.1 degree accurate attitu le determination).

Figures 7 and 8 compar: the steady-state position and velocity performance for the optimistic case with a realisti case starting at the least favorable tracking geonetry and including 0.1 arc-minute directional neasurement biases and Doppler measurements from a noisy USO with Doppler tracking redused to one 2-hour contact every other day (Fealistic with Sun, Earth, and Doppler) and a re:listic case identical to above but without Dopple: tracking (Realistic with Sun and Earth).

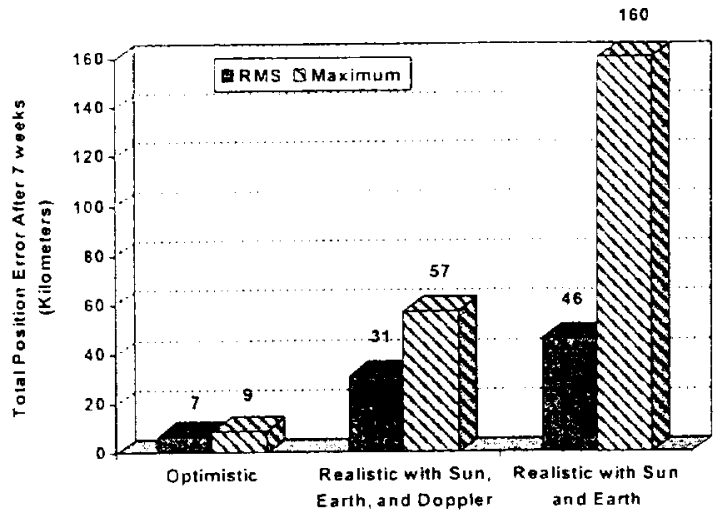

Figure 7: CelNav Position Errors Based on Various Sensor Accuracies

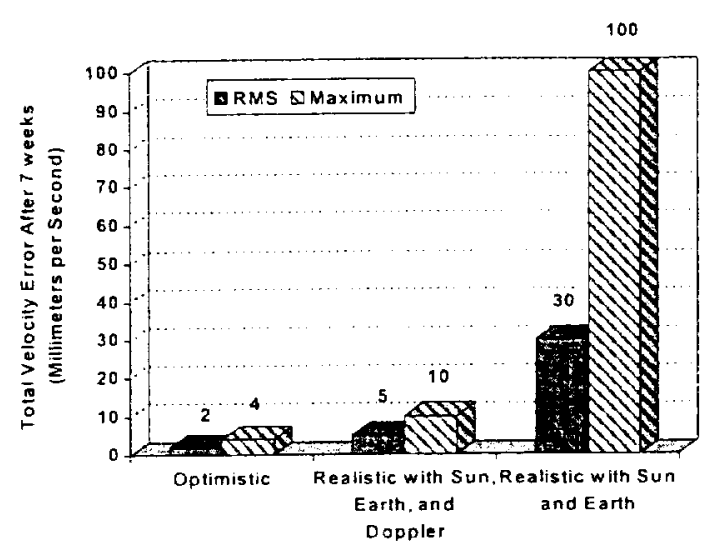

Figure 8: CelNav Velocity Errors Based on Various Sensor Accuracies

As a comparison, analysis using the realistic sensor parameters gave rather good results for a highly elliptical ( 1.8 by $9 \mathrm{Re}$ ) orbit. Attitude sensors alone gave a position RMS of $15 \mathrm{~km}$, while the addition of Doppler data dropped that error to $1.5 \mathrm{~km}$ [Long 00].

\section{Delta Differenced One-Way Range (DDOR)}

All previous LPO missions have used ground based tracking using range and Doppler measurement types. Both of these measurements give information only along the spacecraft lineof-sight. Information perpendicular to this line is inferred only from time-varying changes in these measurements and the dynamical model used. Thus, the radial component of the orbital uncertainty is considerably more accurate than the plane-of-the-sky components. 
DDOR is a true VLBI measu rement type that is being implemented at the $D=34-m$ and $70-\mathrm{m}$ ( $\mathrm{X}$-band only) sites as a no: ninal measurement type by May 2003 [Cangahua:a 01].

DDOR is obtained by duble differencing simultaneous observations of the spacecraft from two widely separated ground sites followed immediately by observations from an angularly nearby quasar (see Figure 9 for a schematic). The differential range to both the spacecraft and the quasar is determined from the observations. These measurements are then algebraically differenced to provide a precise determination of the angular position offset between the two sources as conmon measurement errors tend to cancel. With multiple baselines, the $2 \mathrm{D}$ angular component can be determine 1 . This information provides previously unavailal le plane-of-the-sky knowledge. Potentially, the use of this measurement type could redace plane-of-the-sky orbital uncertainty to the curr nt radial levels.

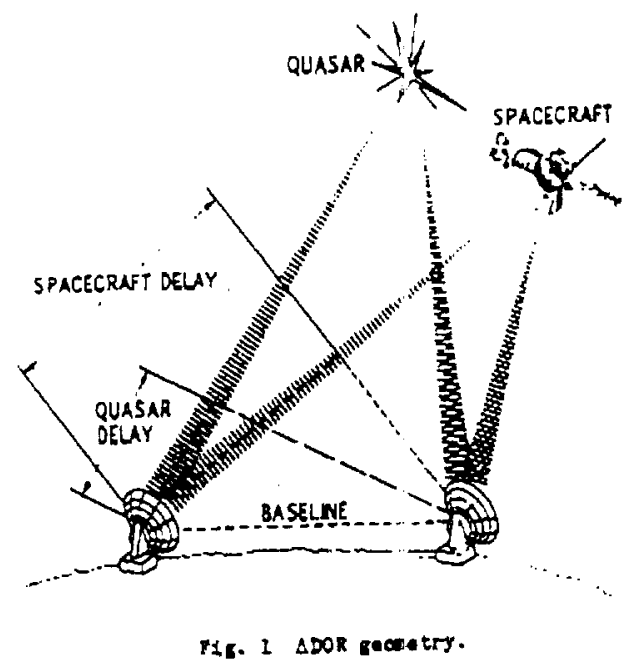

Figure 9: DDOR Measurement Type

Each tracking station simult nneously views the spacecraft and records ridio tones being broadcast. The antennas then simultaneously slew off the spacecraft and record the signals from a reference quasar which is located angularly near the spacecraft The calculation of the angular separation betueen the spacecraft and the quasar is then,

$$
\alpha=\frac{\left(\Delta \tau_{s: C}-\Delta \tau_{j}\right) c}{B}
$$

where $\alpha$ is projection of the angular separation between the spacecraft and quasar onto the baseline between the two stations, $\Delta \tau_{S / C}$ is the time delay between when a radio signal from the spacecraft is received at the first station and when it is received at the second station, $\Delta \tau_{Q}$ is the same for the quasar signal, $c$ is the speed of light, and B is the baseline length [Polimeier 92]. Intercontinental baselines between DSN stations range from 8,000 to $10,500 \mathrm{~km}$. Accuracies are typically expressed as a distance measurement (numerator of above equation) since the baseline lengths vary.

DDOR has been used operationally before on interplanetary missions such as Voyager, Galileo, and Magellan. It has also been tested on Mars Global Surveyor and Mars Odyssey. Accuracies of 21 to $50 \mathrm{~cm}$ were seen for Voyager measurements (30 cm equals $37.5 \mathrm{nrad}$ at $8000 \mathrm{~km}$ baseline) [Border 82]. The DDOR requirement for Galileo was 50 nrad [Pollmeier 92]. Current DDOR implementation states accuracies of 7.5 nrad with telemetry subcarriers and 5 nrad with Differenced One-Way Range tones [Cangahuala 01]. Figure 9 shows the DDOR error budget for the current implementation.

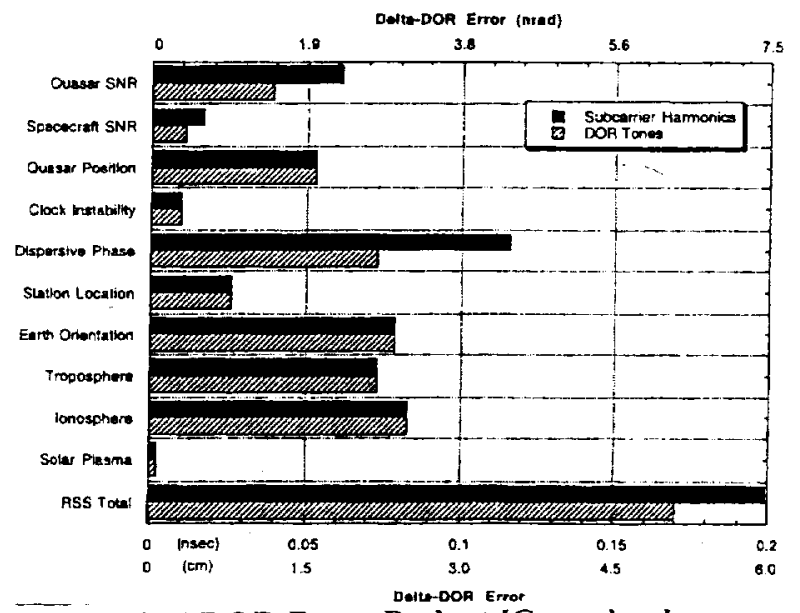

Figure 9: DDOR Error Budget [Cangahuala 01].

Covariance analysis was performed by GNCC at the request of NASA HQ to assess the use of DDOR for LPOs. Analysis indicates improved orbital accuracy can be obtained while reducing tracking times by $80 \%$. Table 10 details some of the analysis for a SOHO orbit using 14 hours per week of DSN rracking. The use of DDOR measurements could reduce that tracking to 2.5 
hours per week and imprive total position uncertainty by more than $25 \%$

Table 10: Covariance Analy sis Results Using DDOR for LJ'Os

\begin{tabular}{|c|c|c|c|c|}
\hline$\frac{\underline{R \& D}}{\operatorname{Sch}^{*}}$ & DDOR & Baseline & $\frac{\frac{\text { Def }}{\text { Pos }}}{\frac{\mathrm{Acc}}{(\mathrm{km})}}$ & $\begin{array}{l}\frac{\text { Tot }}{\mathrm{DSN}} \\
\frac{\text { Trk }}{\text { Time }} \\
\frac{(\mathrm{hrs} /}{\mathrm{wk})} \\
\end{array}$ \\
\hline $\begin{array}{c}2 \\
\text { hrs/day }\end{array}$ & None & $\overline{N A}$ & 3.8 & 14 \\
\hline $\begin{array}{l}1 \mathrm{hr} / 3 \\
\text { days }\end{array}$ & None & $\overline{\mathrm{NA}}$ & 6.5 & 2.5 \\
\hline $\begin{array}{l}1 \mathrm{hr} / 3 \\
\text { days }\end{array}$ & $\begin{array}{c}\text { Once/da } \\
y\end{array}$ & $\begin{array}{c}50 \% \text { Gis- } \\
\text { Mad } \\
12 \% \text { Can- } \\
\text { Mad } \\
38 \% \text { Gis- } \\
\text { Can } \\
\end{array}$ & 2.8 & 2.5 \\
\hline
\end{tabular}

* Rotating stations each day including both northern/southern hemisphere

The use of DDOR has many advantages. DDOR is one-way data type (downink only). There is no need to calibrate the spacecraft uplink for refraction which simplifies ground station operation. Spacecraft angulas position, or planeof-the-sky position components, is more accurately determined by DDOR; five-fold improvements are possible.

However, there are drawbicks to the use of DDOR. For each acquisiticn during Voyager, $10^{9}$ bits were reduced to obtain one measurement. This extensive post-processing typically took up to $24 \mathrm{hrs}$ [Porder 82]. Thus far, DDOR has been used as a supplemental measurement source with in lependent solutions obtained from standard measurement types used as references. An increased reliance on DDOR and large reductions in staniard tracking would reduce the quality of assurance of DDOR.

\section{CONCLUSIONS}

Previous LPO missions have obtained OD accurate to 2 to $10 \mathrm{~km}$. Th. best accuracy has been achieved by MAP and s due in part to the favorable attitude and cons stent $C_{r}$ estimates. The worst accuracy has beell achieved by ACE and is due primarily to the shortening of the batch data arc due to irequent spacecraft perturbations. The amount of tracking data received for each mission is not highly correlated with the $O D$ accuracy achieved. This suggests that other issues such as spacecraft perturbations, spacecraft attitude, and use of MARK IVA data are morc important than quantity of tracking data.

The use of DDOR measurements can increase the accuracy of a standard range and Doppler tracking scenario by $25 \%$ while reducing the total amount of tracking time by $80 \%$. DDOR data greatly inproves the plane-of-the-sky position error components. While DDOR has not yet been used for any LPO missions, it has been used operationally on interplanetary missions.

The use of CelNav would eliminate the need for all coherent dedicated tracking passes. The performance of CelNav using realistic sensor performance indicates that autonomous navigation using directional and Doppler measurements can meet onboard navigation requirements on the order of $30 \mathrm{~km}$. Higher accuracy is achievable by reducing measurement noise and increasing the Doppler tracking frequency. Autonomous navigation using only directional measurements can provide a lowercost navigation method for missions with less stringent onboard navigation requirements, i.e. greater than $50 \mathrm{~km}$.

\section{REFERENCES}

Border, J. S., et al, "Deternining Spacecraft Angular Position with Delta VLBI: The Voyager Demonstration", AIAA-82-1471, AIAA/AAS Astrodynamics Conference, San Diego, CA, Aug 9-11, 1982,

Cangahuala, L. A., "Navigation Measurements: Overview, Performance and Future Plans", Navigation Tracking Requirements Peer Review slides, June 26, 2001.

Colombe, B., SOHO orbit analyst. personal conmunication on SOHO OD strategies, April 2002.

Fink, D., "MAP L2 OD Accuracies Report", internal memorandum dated Apr 17, 2002.

Folta, D., et al, "Autonomous Navigation Using Celestial Objects", AAS 99-439, 1999. 
Jordan, P., et al, "Solar and Heliospheric Observatory ( $\mathrm{SOHO}$ ) Missio 2 Description and Flight Dynamics Analysis Re yorts, Revision 2". CSC/TM-91/6030ROUDO, Stp 1993.

Joyce, J. B., et al, "Traject ory Determination Support and Analysis for ISEE-3 from Halo Orbit to Escape from the Ea th Moon System", AIAA-84-1980, AIAA/AAS Astrodynamics Conference, Seattle. WA, Aug 20-22, 1984.

Long, A., et al, "Autonomnus Navigation of High-Earth Satellites Using Celestial Objects and Doppler Measurements", AIAA 2000-3937, AIAA/AAS Astrodynanics Specialist Conference, Denver, CO, Aug 14-172000.

Marr, G., "Constellatio:I-X L2 Orbit Determination $O D$ Error Analysis with Impulsive Momentum Unlouding Maneuvers", memorandum to Constellation- $\mathrm{X}$ project dated June 5, 1998.

Nicholson, A., "MAP Tracking Assessment for Reduced Volume of Data", Computer Sciences Corporation memorandum dared Sept 10, 1999.

Pollmeier, V. M. \& Kalleme in, P. H., "Galileo Orbit Determination from L aunch through the First Earth Flyby", The Instiute of Navigation, $47^{\text {th }}$ Annual Meeting, 1992. 